\title{
An instrument to quickly and reliably classify aphasic patients' symptoms in syndromes based on cognitive assessments
}

\author{
Maximiliano A. Wilson ${ }^{1}$, Macarena Martínez-Cuitiño ${ }^{2}$ and Yves Joanette ${ }^{1}$ \\ 1- Institut Universitaire de Gériatrie de Montréal, Quebec, Canada \\ 2- Universidad de Buenos Aires, Argentina
}

\begin{abstract}
The classification of aphasic patients' symptoms into a syndrome is a crucial procedure that favours and simplifies the communication among the clinicians involved in patient treatment and follow-up. Nevertheless, the aphasic population usually presents a very heterogeneous symptomatology; consequently, classical syndromes do not offer a good basis to plan their rehabilitation relying on their specific language impairments. Current rehabilitation strategies frequently require the identification of specific cognitive abilities affected. This is why it is common in clinical practice to assess aphasic patients with a syndromic protocol in order to classify them as well as a cognitive battery to plan their rehabilitation. The aim of the present article is to present a quick and reliable tool (i.e., a syndromic classification sheet) to help clinicians classify their clients' symptomatology in a syndrome by means of only one cognitive neuropsychological assessment. Two groups of speech therapists (5 experienced and 5 inexperienced) were asked to classify 5 aphasic patients from a syndromic classification sheet and their assessment protocols. Results indicate an acceptable inter-rater agreement for the experienced group but not for the inexperienced one. In conclusion, the classification sheet turned out to be a useful and reliable tool to classify aphasic patients in a given syndrome and by means of only some subtests from a cognitive assessment battery, provided that clinicians had some years of experience. In addition, this new instrument enables language clinicians to shorten the assessment of their aphasic clients. Keywords: aphasia, cognitive assessment, syndrome, reliability.
\end{abstract}

Received 9 November 2009; received in revised form 26 December 2009; accepted 27 December 2009. Available on line 29 December 2009.

\section{Introduction}

Currently, two main approaches can be distinguished in the domain of aphasia assessment: the classical clinicalneuroanatomical and the cognitive neuropsychological one (Spreen \& Risser, 2003). The first approach attempts to group the symptoms that a given patient presents into a particular aphasic syndrome. For example, if a patient is non-fluent, has phonemic and phonetic paraphasias and mild anomia when speaking or repeating, and maintains relatively unimpaired comprehension, this constellation of symptoms would be classified as Broca's aphasia. On the other hand, the cognitive neuropsychological

Maximiliano A. Wilson, Centre de Recherche de l'Institut Universitaire de Gériatrie de Montréal, Quebec, Canada. Macarena Martínez-Cuitiño, Universidad de Buenos Aires, Argentina. Yves Joanette, PhD, Centre de Recherche de l'Institut Universitaire de gériatrie de Montréal \& Faculté de médecine, Université de Montréal, Quebec, Canada. Correspondence regarding this article should be directed to: Maximiliano A. Wilson, Centre de Recherche, Institut Universitaire de Gériatrie de Montréal, 4565 chemin Queen Mary, Montreal QC H3W 1W5, Canada. Phone: +1-514-340-3540, ext. 4003. Fax: +1-514-340-3548. E-mail: maximiliano.wilson@criugm.qc.ca approach aims at identifying the impaired language components based on cognitive and psycholinguistic models of processing. For instance, the same patient described above would be characterized as having deficits in the phonological output component or short term phonological buffer, depending on his/her pattern of performance.

Historically, the notion of syndromes responded to the need of grouping the symptoms that were regularly found together in groups of patients to infer the possible lesion site in a moment where imaging studies were not available (Dronkers, Redfern, \& Knight, 2000). Then, the definition of the subtypes of aphasia was based on clinical and neuroanatomical data because the lesion site would presumably explain the type of aphasia a patient had (Spreen \& Risser, 2003). Nowadays, with the advent of imaging techniques (such as MRI and PET scans), the idea of a detailed description to infer a possible lesion site has become obsolete (Dronkers, Redfern, \& Knight, 2000). However, the simplicity behind the notion of a syndrome still facilitates the communication among different health professionals who are not specialized in language assessment or rehabilitation (Wilson, 2007). 
Nevertheless, most of the aphasic patients present a constellation of symptoms that cannot be reliably classified in only one particular syndrome (Basso, 2003; Cherney \& Small, 2009; Dronkers \& Ludy, 1998; Dronkers, Redfern, \& Knight, 2000; Spreen \& Risser, 2003; Strauss, Sherman, \& Spreen, 2006). This important heterogeneity of aphasic symptoms in each patient is not taken into account when a patient is "labeled" with a syndrome and constitutes a great disadvantage for the clinicalneuroanatomical approach and for the subsequent rehabilitation planning. This is why many speech therapists also assess their clients with a battery of tests based on the cognitive neuropsychological approach. This type of assessment provides detailed information about the profile of impaired and preserved components of language processing in each individual patient and, thus, helps design a rehabilitation protocol (Basso, 2003; Ellis \& Young, 1988; Spreen \& Risser, 2003). Complementary cognitive assessment tools appear to be essential to the diagnosis and rehabilitation of specific cognitive profiles and their relation to language components processing.

Consequently, in everyday practice, clinicians usually assess their clients who suffer from aphasia with two different batteries: one based on the clinical-neuroanatomical approach that helps them to classify their clients in a given syndrome, and a second assessment based on cognitive neuropsychological batteries to plan their rehabilitation. This leads to redundant and timeconsuming assessment sessions. Thus, the aim of the present article is to present a simple tool to classify the symptomatology of an aphasic patient into a syndrome and to provide evidence of its reliability. The instrument here presented allows clinicians to identify the classical syndromes based on the different cognitive components affected in a given patient in less than ten minutes.

\section{Methods}

\section{Participants}

Two groups of five speech therapists each, from two universities in Buenos Aires, Argentina, participated in the study as raters. They were divided as follows: 1) experienced clinicians (mean years of experience $=11$; range $=3-18$ years); and 2) inexperienced clinicians (mean years of experience $=1$; range $=.25-1.5$ years). Participant's main field of clinical practice was either audiology, voice treatment, or swallowing and dysphagia rehabilitation (Awan \& Roy, 2009; Robbins, Butler, Daniels, \& Diez Gross, 2008) and children language assessment but none of them had previous experience in conducting adult language speech therapy.

\section{Instruments}

The procedure relies on a simple 6-question profile that can be answered by looking at any cognitive assessment protocol and enables the syndromic categorization. All the participants were given:

1) A classification sheet, that included a questionnaire with six simple yes-no questions (see Appendix A). It surveyed the main tasks (auditory comprehension, repetition, picture naming, and spontaneous speech) and symptoms (fluency, paraphasias, and anomias) that have helped classify aphasic patients in the seven classical syndromic profiles (Goodglass \& Kaplan, 1996). The classical syndromic profiles were also provided in the classification sheet.

2) The assessment protocols and scores of five aphasic patients. The patients were assessed by means of the BEA Battery (Battery for the Assessment of Aphasia; Wilson, 2009; Wilson, Jaichenco, \& Ferreres, 2005), a cognitive language assessment battery. All the patients had mild to moderate aphasia due to a single cerebrovascular accident (CVA). They were assessed more than six months post-CVA onset. All participants were former patients at the neuropsychological and speech pathology units in three different hospitals in Buenos Aires, Argentina. They were specifically called to participate in the present study and their family members gave consent to participation in the study. They were not receiving any speech language treatment at the moment of the assessment because they were neurologically stable.

3) Recordings of oral production of the same five aphasic patients. The patients were asked to tell the story of "Little Red Riding Hood" so that three to five minutes of their oral production could be recorded.

\section{Procedure}

Each participant was presented with one copy of the materials. Each group of speech therapists performed their classification in a separate session: one for the experienced and another for the inexperienced therapists. The session lasted 50 minutes in total. They were asked to individually follow the instructions provided in the classification sheet (Appendix A), to observe each patient's assessment results and to answer the six yes-no questions. Also, they were provided with written instructions that explained them which subtests from the BEA Battery they should look at in order to answer each question. In order to answer whether the patients were fluent or not (question 3) participants listened to the patients' oral production recordings. Afterwards, and following the pattern of yes-no answers, they were asked to classify each patient in a given syndrome by comparing the patients' profiles to those of the classical syndromes (based on Goodglass \& Kaplan, 1996). For that 
purpose, they had to write the name of the syndrome they thought adequate for each patient under his/her column. They were asked not to leave any unanswered questions and no feedback was given as to the correct answers during the classification procedure. During the entire procedure participants were not allowed to consult their peers about the answers and requested to keep them strictly individual.

\section{Statistics}

In order to calculate the agreement observed among the independent observers we used the Krippendorff's Alpha (Hayes \& Krippendorff, 2007) for each group of participants separately. Among its advantages, this measure is suited for nominal scales of measurement (as in the present case) and it can be easily calculated and interpreted: its SPSS output provides a numerical scale that can take values from 0 (absence of agreement) to 1 (perfect agreement), being the agreement value larger when it is closer to 1, as in Cronbach's Alpha.

\section{Results}

In the group of experienced speech therapists the inter-rater agreement obtained was acceptable (Krippendorff's $\alpha=.66$ ). Contrarily, the agreement among the inexperienced speech therapists was low (Krippendorff's $\alpha=.29$ ). It is noteworthy that the alpha value for the experienced group becomes even more considerable if it is taken into account that each rater had to choose among seven possible syndromes, which makes the agreement even harder. Figure 1 shows the agreement (in percentage) for each patient (1-5) as a function of clinical experience of the groups (experienced vs. inexperienced).

Figure 1 shows that the mean agreement for the experienced group of judges is clustered in the upper part of the $\mathrm{x}$-axis, suggesting larger agreement as compared to the inexperienced group, in which the agreement clusters closer

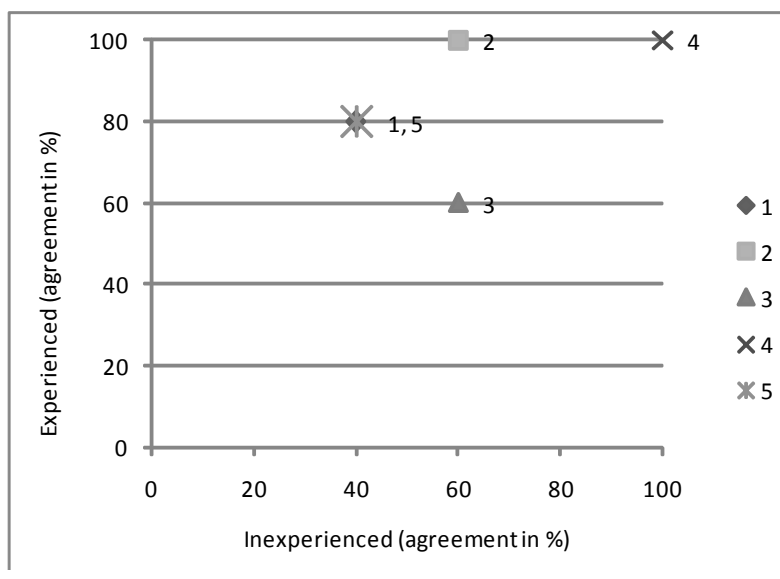

Figure 1. Agreement (in \%) for each patient (1-5) as a function of the clinical experience of the groups: experienced vs. inexperienced. to the y-axis. Then, as a general trend, agreement tends to be greater for the experienced speech therapists. However, and notably, when a patient presents a prototypical pattern of symptoms that can be unequivocally labeled as only one syndrome, as it is the case of patient 4, a typical Broca's aphasic, then the agreement is perfect, even among the inexperienced judges.

On the other hand, if we consider the amount of syndrome choices that participants made, we can see that the experienced group had a mean of 1.6 choices per patient (range $=1-2$ ) and the inexperienced group had a mean of 2.6 choices (range $=1-4$ ). That is to say, the experienced group not only agreed more on a given syndrome for a given patient but had fairly less distributed non-agreements as compared to the inexperienced group of speech therapists.

\section{Discussion}

Nowadays, the classical syndromic classification has turned towards a more flexible and mostly descriptive conceptualization, taking into account the difficulty to reliably classify the aphasic patient population (Basso, 2003). Nevertheless, the simplicity of a syndromic label still remains as a great advantage to communicate with other professionals involved in assessment, follow-up or rehabilitation of aphasic patients.

However, and given that often the assessment sessions are limited by health providers, they should be devoted to obtain valuable information to plan rehabilitation. In that regard, a comprehensive cognitive assessment is the best available tool to plan individual rehabilitation on the bases of particularities and heterogeneity of symptoms. That is why the aim of the present article was to provide language clinicians and speech therapists with a friendly and time-saving instrument to enable them to reliably classify an aphasic patient by taking into consideration only the data gathered from a cognitive assessment.

In general, it can be stated that the classification difficulties for the inexperienced group of speech therapists reproduced the pattern found in clinical literature (Spreen \& Risser, 2003) as well as in neuroimaging studies (Dronkers, Redfern \& Knight, 2000). It seems that to classify aphasic symptoms into syndromes theoretical knowledge is not sufficient when clinicians lack general clinical experience in speech therapy.

Conversely, the classification sheet turned out to be a useful and reliable tool to classify aphasic patients in a given syndrome and by means of only some subtests from a cognitive assessment battery, provided that clinicians had some years of experience. It can be argued that the experienced clinicians had greater expertise to assess aphasic patients as compared to the inexperienced speech therapists. However, their professional field of 
practice was not related to language deficit assessment or rehabilitation. This suggests that experience indeed favored clinical judgment but its nature is general rather than focused on language assessment. By choosing all of the clinicians from a non-language assessment clinical field, the most difficult scenario was given for them to rate the patients' language deficit. In such a way, we believe that stronger evidence was provided for the reliability of the instrument presented here.

In sum, this classification sheet will enable language clinicians to avoid the syndromic assessment of aphasic patients in order to identify and classify them in a classical syndrome. Even more, they will be able to shorten the assessment sessions only to a cognitive approach assessment that will help them plan the rehabilitation of their patients suffering from aphasia.

Finally, this study has been conducted in Spanish and with a cognitive battery for Spanish-speaking aphasic patients. We believe that these results will also apply to any cognitive assessment battery, irrespectively of the language. Nevertheless, further testing in equivalent versions of the syndromic sheet in different languages should be carried out. Then, caution is advisable when applying the classification sheet presented here in languages other than Spanish.

\section{Acknowledgments}

Thanks to FrancineGiroux from theCentrede Recherche de l'Institut Universitaire de Gériatrie de Montréal for her help in the choice of the data analysis test. Also, our gratitude goes to the patients and speech therapists that so willingly accepted to participate in this study.

\section{References}

Awan, S., \& Roy, N. (2009). Outcomes Measurement in Voice Disorders: Application of an Acoustic Index of Dysphonia Severity. Journal of Speech, Language, and Hearing Research, 52, 482-499.

Basso, A. (2003). Aphasia and its therapy. New York: Oxford University.

Cherney, L.R., \& Small, S.L. (2009). Aphasia, apraxia of speech, and dysarthria. In: Stein, J., Harvey, R.L., Macko, R.F., Winstein, C.J., \& Zorowitz, R.D. (Eds.), Stroke recovery and rehabilitation (pp. 155-182). New York: Demos Medical.

Dronkers, N.F., \& Ludy, C.A. (1998). Brain lesion analysis in clinical research. In: Stemmer, B., \& Whitaker, H. (Eds.), Handbook of Neurolinguistics (pp. 174-188). New York: Academic Press.

Dronkers, N.F., Redfern, B., \& Knight, R. (2000). The neural architecture of language disorders. In: Gazzaniga, M.S. (Ed.), The New Cognitive Neurosciences (pp. 949-958). Cambridge, MA: MIT Press.

Ellis, A., \& Young, A. (1988). Human Cognitive Neuropsychology: A Textbook with Readings. Hove, UK: Psychology Press.

Goodglass, H., \& Kaplan, E. (1996). Evaluación de la afasia y de trastornos relacionados. Madrid: Panamericana.

Hayes, A., \& Krippendorff, K. (2007). Answering the call for a standard reliability measure for coding data. Communication Methods and Measures, 1, 77-89.

Robbins, J., Butler, S., Daniels, S., \& Diez Gross, R. (2008). Swallowing and Dysphagia Rehabilitation: Translating Principles of Neural Plasticity Into Clinically Oriented Evidence. Journal of Speech, Language, and Hearing Research, 51, 276-301.

Spreen, O., \& Risser, A. (2003). Assessment of Aphasia. New York: Oxford University.

Strauss, E., Sherman, E., \& Spreen, O. (2006). A compendium of neuropsychological tests. New York: Oxford University.

Wilson, M. (2007). Enfoques Neuropsicológicos en la evaluación de la Afasia: Revisión de los instrumentos en español. In: Alchieri, J. (Ed.), Avaliação psicológica: perspectivas e contextos (pp. 47-66). Brazil: Vetor.

Wilson, M. (2009). Evaluación de los trastornos del lenguaje. Presentación de una batería clínica con fundamentación cognitiva (Unpublished Doctoral dissertation). Department of Medicine, University of Buenos Aires, Argentina.

Wilson, M., Jaichenco, V., \& Ferreres, A. (2005). Batería de Evaluación de la Afasia (BEA) basada en modelos neuropsicolingüísticos [Abstract]. Actas del VII Simposio de Psicolingüística, 7, 53. 
Appendix A - Syndromic classification sheet

Surname:

Experience (in years):

Instructions:

Below you will find a grid in which you have to rate 5 aphasic patients. For each patient, you have to complete 6 YES-NO questions, based on your judgment and on the assessment information available for each patient. In some cases the answer will not be easy (doubtful YES or NO), but you have to choose the one that best suits your answer, in EVERY CASE.

\begin{tabular}{|c|c|c|c|c|c|c|c|c|c|c|c|}
\hline & & \multicolumn{10}{|c|}{ Patient } \\
\hline & & \multicolumn{2}{|c|}{1} & \multicolumn{2}{|c|}{2} & \multicolumn{2}{|c|}{3} & \multicolumn{2}{|c|}{4} & \multicolumn{2}{|c|}{5} \\
\hline & & Yes & No & Yes & No & Yes & No & Yes & No & Yes & No \\
\hline 1 & Does the patient understand? & & & & & & & & & & \\
\hline 2 & Does the patient repeat correctly? & & & & & & & & & & \\
\hline 3 & Is the patient fluent? & & & & & & & & & & \\
\hline 4 & $\begin{array}{l}\text { Does the patient have severe ano- } \\
\text { mias? }\end{array}$ & & & & & & & & & & \\
\hline 5 & Has he got severe paraphasias? & & & & & & & & & & \\
\hline 6 & $\begin{array}{l}\text { Has the patient got lexical parapha- } \\
\text { sias? }\end{array}$ & & & & & & & & & & \\
\hline & Aphasic syndrome & & & & & & & & & & \\
\hline
\end{tabular}

In order to answer the questions above, the following table indicates the tests from the assessment sheet you should look at to obtain the adequate information:

\begin{tabular}{lll} 
& Question & Test \\
\hline 1 & Understands? & Auditory comprehension of objects and actions \\
\hline 2 & Repeats? & Word repetition \\
\hline 3 & Fluent? & Oral production (recordings) \\
\hline 4 & Severe anomia? & Oral picture naming \\
\hline 5 & Severe paraphasias? & Oral picture naming \\
& & Word repetition \\
\hline 6 & Lexical paraphasias? & Oral picture naming \\
\hline
\end{tabular}




\section{Appendix A - Cont.}

Syndromic profiles

In the following grids the 7 classical syndromic profiles are shown. Once you have completed the syndromic classification sheet, please compare the profile of each of the 5 aphasic patients to those expected for each syndrome. For each patient choose the syndrome that best fits the profile obtained by the patients.

It is very likely that some of the patients' profiles will NOT MATCH exactly any syndromic profile. If that is the case, please choose the profile that best matches one of the syndromic profiles. Finally, write the syndrome name in the space provided to that end, after the 6 questions.
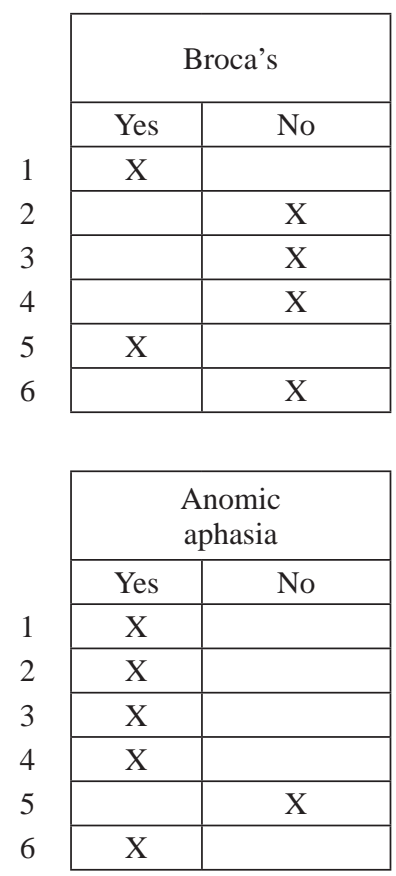
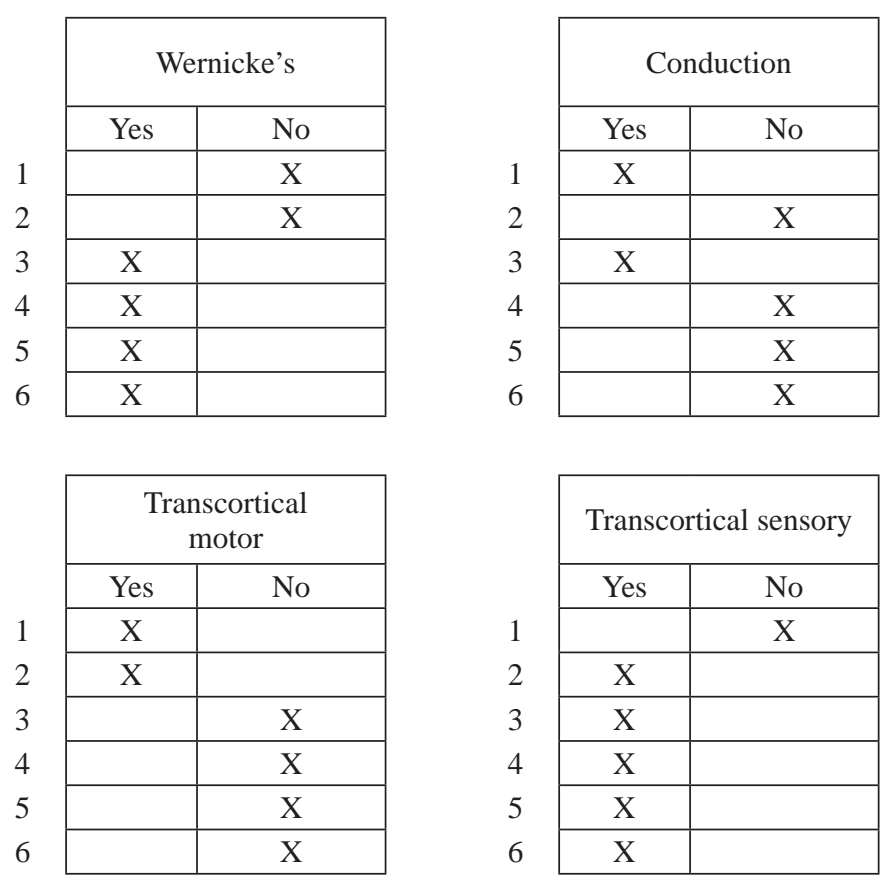

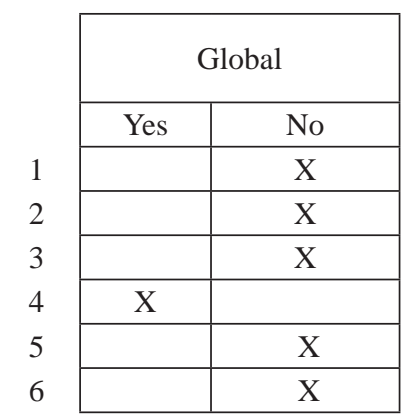

